\title{
A Modified Thakur Three-Step Iterative Algorithm to Garcia-Falset Mappings and Variational Inequalities
}

\author{
Xinhong Chen ${ }^{1}$, Wenxiang Han², Liping Gong ${ }^{3}$, Chengjun Luo ${ }^{3}$ \\ ${ }^{1}$ College of Science, Zhongyuan University of Technology, Zhengzhou, China \\ ${ }^{2}$ School of Civil Engineering, Zhongyuan University of Technology, Zhengzhou, China \\ ${ }^{3}$ School of Foreign Languages, Zhongyuan University of Technology, Zhengzhou, China \\ Email: xinhongchen11@163.com
}

How to cite this paper: Chen, X.H., Han, W.X., Gong, L.P. and Luo, C.J. (2020) A Modified Thakur Three-Step Iterative Algorithm to Garcia-Falset Mappings and Variational Inequalities. Journal of Applied Mathematics and Physics, 8, 2930-2942. https://doi.org/10.4236/jamp.2020.812216

Received: November 16, 2020 Accepted: December 18, 2020 Published: December 21, 2020

Copyright $\odot 2020$ by author(s) and Scientific Research Publishing Inc. This work is licensed under the Creative Commons Attribution International License (CC BY 4.0).

http://creativecommons.org/licenses/by/4.0/

\begin{abstract}
In this paper, we suggest and analyze a modified Thakur three-step iterative algorithm to approximate a common element of the set of common fixed points of Garcia-Falset mappings and the set of solutions of some variational inequalities in Banach spaces. We also establish strong convergence theorems for a common solution of the above-said problems by the proposed iterative algorithm without the compactness assumption. The methods in this paper are novel and different from those given in many other papers. And the results are the extension and improvement of the recent results announced by many others.
\end{abstract}

\section{Keywords}

Banach Space, Fixed Point, Garcia-Falset Mapping, Strong Convergence, Variational Inequality

\section{Introduction}

In the early 1960s, Stampacchia [1] introduced the variational inequalities theory, which has emerged as an interesting branch of applied mathematics with a wide range of applications in industry, physics, optimization, social and science. The variational inequalities are closely related with many general problems of Nonlinear Analysis, such as fixed point, complementarity and optimization problems. It has been extended and generalized in several directions using novel and new techniques.

On the other hand, the theory of fixed points has become one of the very po- 
werful tools of nonlinear analysis. Further, by the development of accurate and efficient techniques for computing fixed points, the effectiveness of the concept for applications has been increased enormously. In recent years, the theory of fixed points has grown rapidly into a flourishing and dynamic field of study both in pure and applied mathematics. It has become one of the most essential tools in the study of nonlinear phenomena. The iterative methods for approximating fixed points are of great importance for modern numerical mathematics (see, e.g., [2] [3] [4] [5]).

The study for variational inequalities, fixed points and approximation algorithms became a topic of intensive research efforts in recent years. Nowadays, this is still one of the most active fields in mathematics. Meanwhile, the nature of many practical problems arouses an iterative approach to the solution. Recently, Garcia-Falset et al. [6] introduced a new class of mappings satisfying the so-called condition (E) (in the sequel, the class of mappings satisfying condition (E) will be referred to as Garcia-Falset mappings). The class of Garcia-Falset mappings covers the class of Suzuki mappings and nonexpansive mappings. However it is still included by quasi-nonexpansiveness. The study for Garcia-Falset mappings with iterative processes using a Banach space as underlying setting is only at the beginning (more precisely; there are just two research papers on uniformly convex Banach spaces that connect mappings endowed with property (E) (see [7] [8]). In order to establish strong convergence results for approximation of fixed points of Garcia-Falset mappings in Banach space, Gabriela et al. [7] and Houmani and Turcanu [8] adopt different iteration schemes, respectively. However, the compactness assumption imposed on $C$ is indispensable in both two papers.

Motivated and inspired by the work in the literature, we suggest and analyze a modified Thakur three-step iterative algorithm to approximate a common element of the set of common fixed points of Garcia-Falset mappings and the set of solutions of some variational inequalities in Banach spaces. We also establish strong convergence theorems for a common solution of the above-said problems by the proposed iterative algorithm without the compactness assumption. The methods in this paper are novel and different from those given in many other papers. And the results are the extension and improvement of the recent results in the literature; see [9] [10] [11] [12].

\section{Preliminaries}

Throughout this paper, we assume that $E$ is a real Banach space with a dual $E^{*}$, $\mathbb{R}$ is the set of real numbers, $\langle\cdot, \cdot\rangle$ is the generalized duality pairing between $E$ and $E^{*}, I$ is the identity mapping on $E$, and $\mathbb{N}$ is the set of nonnegative integers. We denote by $x_{n} \rightarrow x$ and $x_{n} \rightarrow x$ the strong and weak convergence of the sequence $\left\{x_{n}\right\}$, respectively. And $\omega_{w}\left(x_{n}\right)$ denote the set of weak limit points of the sequence $\left\{x_{n}\right\}$. The set of fixed points of $T: C \rightarrow C$ is denoted by $\operatorname{Fix}(T)$. The (normalized) duality mapping of $E$ is denoted by $J$, that is, 


$$
J(x)=\left\{x^{*} \in E^{*}:\left\langle x, x^{*}\right\rangle=\|x\|^{2},\left\|x^{*}\right\|=\|x\|\right\}
$$

for all $x \in E$. If $E$ is a Hilbert space, then $J=I$, where $I$ is the identity mapping.

A Banach space $E$ is said to be smooth if the limit

$$
\lim _{t \rightarrow 0} \frac{\|x+t y\|-\|x\|}{t}
$$

exists for all $x, y$ on the unit sphere $S(E)=\{x \in E:\|x\|=1\}$.

Assume $\varphi$ defined $\mathbb{R}^{+}:=[0, \infty)$ is a continuous strictly increasing function such that $\varphi(0)=0$ and $\lim _{r \rightarrow \infty} \varphi(r)=\infty$. This function $\varphi$ is called a gauge function. The duality mapping $J_{\varphi}: E \rightarrow 2^{E^{*}}$ defined by

$$
J_{\varphi}(x)=\left\{x^{*} \in E^{*}:\left\langle x, x^{*}\right\rangle=\|x\| \varphi(\|x\|) \text { and }\left\|x^{*}\right\|=\varphi(\|x\|)\right\} .
$$

In the case that $\varphi(t)=t, J_{\varphi}=J$, where $J$ is the normalized duality mapping. Clearly, the relation $J_{\varphi}(x)=\frac{\varphi(\|x\|)}{\|x\|} J(x), \forall x \neq 0$ holds (see, e.g., [13]). Browder [14] initiated the study $J_{\varphi}$. Set, for $t \geq 0$,

$$
\Phi(t)=\int_{0}^{t} \varphi(r) \mathrm{d} r
$$

As we know that $J_{\varphi}(x)$ is the subdifferential of the convex function $\Phi(\|\cdot\|)$ at $x$. Following Browder [14], we say that Banach space $E$ has a weakly continuous duality map if there exists a gauge $\varphi$ such that the duality map $J_{\varphi}$ is single-valued and continuous from $E$ with the weak topology to $E^{*}$ with weak ${ }^{*}$ topology. Every $l^{p}(1<p<\infty)$ space has a weakly continuous duality map with the gauge $\varphi(t)=t^{p-1}$ (see, e.g., [15] [16]). A space with a weakly continuous duality map is easily seen to satisfy Opial's condition (see [14]). Conversely, if Banach space satisfies Opial's condition and has a uniformly Gâteaux differentiable norm, then it has a weakly continuous duality mapping.

Remark 2.1. It is well known that $J_{\varphi}$ is single-valued if and only if $(E,\|\cdot\|)$ is smooth (see, e.g., [17]).

Let $E$ be a real Banach space, $C$ a nonempty closed convex subset of $E, T$ a mapping on $C$ and $F(T):=\{x \in C: T x=x\}$.

Definition 2.1. A mapping $T: C \rightarrow C$ is said to be:

1) Contractive if there exists a constant $\alpha \in(0,1)$ such that

$$
\|T x-T y\| \leq \alpha\|x-y\|, \forall x, y \in C ;
$$

2) Nonexpansive if $\|T x-T y\| \leq\|x-y\|$ for all $x, y \in C$.

3) Quasinonexpansive if $\left\|T x-x^{*}\right\| \leq\left\|x-x^{*}\right\|, \forall x \in C, x^{*} \in F(T)$.

Definition 2.2. A mapping $T: C \rightarrow E$ is said to satisfy condition ( $C$ ) on $C$ if for all $x, y \in C$ with $\|T x-x\| \leq 2\|x-y\|$, one has that $\|T x-T y\| \leq\|x-y\|$.

Recently, Garcia-Falset et al. [6] introduced a new class of mappings satisfying the so-called condition $(E)$ (in the sequel, the class of mappings satisfying condition $(E)$ will be referred to as Garcia-Falset-generalized nonexpansive mappings or Garcia-Falset mappings). 
Definition 2.3. Let $C$ be a nonempty subset of a Banach space $E$ and $\mu \geq 1 . A$ mapping $T: C \rightarrow E$ which satisfies the inequality

$$
\|x-T y\| \leq \mu\|x-T x\|+\|x-y\|, \forall x, y \in C ;
$$

is said to be endowed with $\left(E_{\mu}\right)$-property. Moreover, we say that T satisfies condition $(E)$ on $C$, whenever $T$ satisfies condition $\left(E_{\mu}\right)$, for some $\mu \geq 1$.

Clearly, condition $(E)$ is weaker than condition $(C)$.

Lemma 2.4. [6] Let $E$ be a real Banach space with the Opial condition. Let $C$ be a nonempty closed convex subset of $E$ and $T: C \rightarrow E$ be a mapping satisfying condition $(E)$ on $C$. Then $T$ is demiclosed at zero, i.e., for any sequence $\left\{x_{n}\right\} \subset C$, if $x_{n} \rightarrow y$ and $\left\|(I-T) x_{n}\right\| \rightarrow 0$, then $(I-T) y=0$.

Recall that, if $C$ and $D$ are nonempty subsets of a Banach space $E$ such that $C$ is closed convex and $D \subset C$, then a mapping $Q: C \rightarrow D$ is sunny [4] provided

$$
P(x+t(x-P(x)))=P(x)
$$

for all $x \in C$ and $t \geq 0$, whenever $P x+t(x-P(x)) \in C$. A mapping $P: C \rightarrow D$ is called a retraction if $P x=x$ for all $x \in D$. Furthermore, $P$ is a sunny nonexpansive retraction from $C$ onto $D$ if $P$ is retraction from $C$ onto $D$ which is also sunny and nonexpansive. A subset $D$ of $C$ is called a sunny nonexpansive retraction of $C$ if there exists a sunny nonexpansive retraction from $C$ onto $D$. The following lemma collects some properties of the sunny nonexpansive retraction.

Lemma 2.5. [18] [19] Let $C$ be a closed convex subset of a smooth Banach space $E$. Let $D$ be a nonempty subset of $C$. Let $P: C \rightarrow D$ be a retraction and let $j$ be the normalized duality mapping and generalized duality mapping on $E$, respectively. Then the following are equivalent.

1) $P$ is sunny and nonexpansive;

2) $\|P x-P y\|^{2} \leq\langle x-y, j(P x-P y)\rangle, \forall x, y \in C$;

3) $\langle x-P x, j(y-P x)\rangle \leq 0, \forall x \in C, y \in D$.

Lemma 2.6. [20] Let $E$ be a uniformly convex Banach space, $r>0$ a positive number, and $B_{r}(0)$ a closed ball of $E$. Then, for any given subset $\left\{x_{1}, x_{2}, \cdots, x_{N}\right\} \subset B_{r}(0)$ and for any positive numbers $\left\{\lambda_{1}, \lambda_{2}, \cdots, \lambda_{N}\right\}$ with $\sum_{i=1}^{N} \lambda_{i}=1$, there exists a continuous, strictly increasing, and convex function $g:[0,2 r] \rightarrow[0, \infty)$ with $g(0)=0$ such that, for any $i, j \in\{1,2, \cdots, N\}$ with $i<j$,

$$
\left\|\sum_{n=1}^{N} \lambda_{n} x_{n}\right\|^{2} \leq \sum_{n=1}^{N} \lambda_{n}\|x\|^{2}-\lambda_{i} \lambda_{j} g\left(\left\|x_{i}-x_{j}\right\|\right) .
$$

Lemma 2.7. [21] Let $\left\{s_{n}\right\}$ be a sequence of nonnegative real numbers, let $\left\{\alpha_{n}\right\}$ be a sequence of $[0,1]$ with $\sum_{n=1}^{\infty} \alpha_{n}=\infty$, let $\left\{\beta_{n}\right\}$ be a sequence of nonnegative real numbers with $\sum_{n=1}^{\infty} \beta_{n}<\infty$, and let $\left\{\gamma_{n}\right\}$ be a sequence of real numbers with $\limsup _{n \rightarrow \infty} \gamma_{n} \leq 0$. Suppose that

$$
s_{n+1} \leq\left(1-\alpha_{n}\right) s_{n}+\alpha_{n} \gamma_{n}+\beta_{n}, \quad n \in \mathbb{N}
$$


Then, $\lim _{n \rightarrow \infty} s_{n}=0$.

Lemma 2.8. [22] Let $\left\{w_{n}\right\}$ be a sequence of real numbers such that there exists a subsequence $\left\{n_{k}\right\}$ of $\{n\}$ which satisfies $w_{n_{k}}<w_{n_{k}+1}$ for all $k \in \mathbb{N}$. Also consider the sequence of positive integers $\{\tau(n)\}$ defined by

$$
\tau(n):=\max \left\{k \leq n: w_{n_{k}}<w_{n_{k}+1}\right\}
$$

for all $n \geq n_{0}$ (for some $n_{0}$ large enough). Then $\{\tau(n)\}$ is a nondecreasing sequence such that $\lim _{n \rightarrow \infty} \tau(n)=\infty$ and it holds that

$$
\max \left\{w_{\tau(n)}, w_{n}\right\} \leq w_{\tau(n)+1} .
$$

\section{Main Results}

Theorem 3.1. Let $C$ be a nonempty closed convex subset of a uniformly convex and smooth Banach space $E$ which admits a weakly continuous duality mapping J. Let $f: C \rightarrow C$ be a contractive mapping with constant $r \in(0,1)$, $T: C \rightarrow C$ be a mapping satisfying condition $(E)$ with $\operatorname{Fix}(T) \neq \varnothing$. For arbitrarily given $x_{0} \in C$, let $\left\{x_{n}\right\}$ be the sequence generated iteratively by.

$$
\left\{\begin{array}{l}
z_{n}=\left(1-\gamma_{n}\right) x_{n}+\gamma_{n} T x_{n}, \\
y_{n}=\left(1-\beta_{n}\right) x_{n}+\beta_{n} T x_{n}, \\
x_{n+1}=\alpha_{n} f x_{n}+\delta_{n} T z_{n}+\eta_{n} T y_{n}, \quad \forall n \in \mathbb{N},
\end{array}\right.
$$

where $\left\{\alpha_{n}\right\},\left\{\beta_{n}\right\},\left\{\gamma_{n}\right\},\left\{\delta_{n}\right\}$ and $\left\{\eta_{n}\right\}$ are real number sequences in $[0$, 1] satisfying.

1) $\lim _{n \rightarrow \infty} \alpha_{n}=0$ and $\sum_{n=1}^{\infty} \alpha_{n}=\infty$,

2) $\alpha_{n}+\delta_{n}+\eta_{n}=1$

3) $0<\liminf _{n \rightarrow \infty} \delta_{n} \leq \limsup _{n \rightarrow \infty} \delta_{n}<1, \quad 0<\liminf _{n \rightarrow \infty} \gamma_{n} \leq \limsup _{n \rightarrow \infty} \gamma_{n}<1$ and $0<\liminf _{n \rightarrow \infty} \eta_{n} \leq \limsup _{n \rightarrow \infty} \eta_{n}<1$.

Then the sequence $\left\{x_{n}\right\}$ converges strongly to a point $p \in \operatorname{Fix}(T)$, which is also the unique solution of the hierarchical variational inequality

$$
\langle f(p)-p, j(q-p)\rangle \leq 0, \forall q \in \operatorname{Fix}(T) .
$$

In other words, $p$ is the unique fixed point of the mapping $P_{\mathrm{Fix}(T)} f$, that is, $p=P_{\mathrm{Fix}(T)} f(p)$.

Proof. We divide the proof into two steps.

Step 1. Firstly, we prove that the sequence $\left\{x_{n}\right\}$ is bounded. Taking $x^{*} \in \operatorname{Fix}(T)$ arbitrarily, it follows by Definition 2.3 that $T$ is quasi-nonexpansive. Hence, we get from (3.1) that

$$
\begin{aligned}
\left\|y_{n}-x^{*}\right\| & =\left\|\left(1-\beta_{n}\right) x_{n}+\beta_{n} T x_{n}-x^{*}\right\| \\
& \leq\left(1-\beta_{n}\right)\left\|x_{n}-x^{*}\right\|+\beta_{n}\left\|T x_{n}-x^{*}\right\| \\
& \leq\left(1-\beta_{n}\right)\left\|x_{n}-x^{*}\right\|+\beta_{n}\left\|x_{n}-x^{*}\right\| \\
& \leq\left\|x_{n}-x^{*}\right\| .
\end{aligned}
$$

In the same way, we get that 


$$
\begin{aligned}
\left\|z_{n}-x^{*}\right\| & =\left\|\left(1-\gamma_{n}\right) x_{n}+\gamma_{n} T x_{n}\right\| \\
& \leq\left(1-\gamma_{n}\right)\left\|x_{n}-x^{*}\right\|+\gamma_{n}\left\|T x_{n}-x^{*}\right\| \\
& \leq\left(1-\gamma_{n}\right)\left\|x_{n}-x^{*}\right\|+\gamma_{n}\left\|x_{n}-x^{*}\right\| \\
& \leq\left\|x_{n}-x^{*}\right\| .
\end{aligned}
$$

It follows from (3.1), (3.2) and (3.3) that

$$
\begin{aligned}
& \left\|x_{n+1}-x^{*}\right\| \\
& =\left\|\alpha_{n} f x_{n}+\delta_{n} T z_{n}+\eta_{n} T y_{n}-x^{*}\right\| \\
& \leq \alpha_{n}\left\|f x_{n}-x^{*}\right\|+\delta_{n}\left\|T z_{n}-x^{*}\right\|+\eta_{n}\left\|T y_{n}-x^{*}\right\| \\
& \leq \alpha_{n}\left\|f x_{n}-f\left(x^{*}\right)\right\|+\alpha_{n}\left\|f\left(x^{*}\right)-x^{*}\right\|+\delta_{n}\left\|T z_{n}-x^{*}\right\|+\eta_{n}\left\|T y_{n}-x^{*}\right\| \\
& \leq \alpha_{n} r\left\|x_{n}-x^{*}\right\|+\alpha_{n}\left\|f\left(x^{*}\right)-x^{*}\right\|+\delta_{n}\left\|z_{n}-x^{*}\right\|+\eta_{n}\left\|y_{n}-x^{*}\right\| \\
& \leq \alpha_{n} r\left\|x_{n}-x^{*}\right\|+\alpha_{n}\left\|f\left(x^{*}\right)-x^{*}\right\|+\delta_{n}\left\|x_{n}-x^{*}\right\|+\eta_{n}\left\|x_{n}-x^{*}\right\| \\
& =\alpha_{n}\left\|f\left(x^{*}\right)-x^{*}\right\|+\left(1-(1-r) \alpha_{n}\right)\left\|x_{n}-x^{*}\right\| \\
& \leq \max \left\{\frac{\left\|f\left(x^{*}\right)-x^{*}\right\|}{1-r},\left\|x_{0}-x^{*}\right\|\right\} .
\end{aligned}
$$

This implies that the sequence $\left\{x_{n}\right\}$ is bounded, so are $\left\{y_{n}\right\}$ and $\left\{z_{n}\right\}$.

Step 2. We show that $\lim _{n \rightarrow \infty}\left\|x_{n}-p\right\|=0$. Here again $p \in \operatorname{Fix}(T)$, which is also the unique solution of the hierarchical variational inequality

$$
\langle f(p)-p, q-p\rangle \leq 0, \quad \forall q \in \operatorname{Fix}(T) .
$$

We analyze this step by considering the following two cases.

Case A: Put $\Gamma_{n}=\left\|x_{n}-p\right\|$ for all $n \in \mathbb{N}$ and assume that $\Gamma_{n+1} \leq \Gamma_{n}$ for all $n \geq n_{0}$ (for $n_{0}$ large enough). In this case, it is easily seen that the $\lim _{n \rightarrow \infty} \Gamma_{n}$ exists. Now we prove that

$$
\lim _{n \rightarrow \infty}\left\|T x_{n}-x_{n}\right\|=0
$$

To see this, we apply Lemma 2.6 to (3.1) to get

$$
\begin{aligned}
& \left\|x_{n+1}-p\right\|^{2} \\
& =\left\|\alpha_{n} f x_{n}+\delta_{n} T z_{n}+\eta_{n} T y_{n}-p\right\|^{2} \\
& \leq \alpha_{n}\left\|f x_{n}-p\right\|^{2}+\delta_{n}\left\|T z_{n}-p\right\|^{2}+\eta_{n}\left\|T y_{n}-p\right\|^{2} \\
& \leq \alpha_{n}\left\|f x_{n}-p\right\|^{2}+\delta_{n}\left\|z_{n}-p\right\|^{2}+\eta_{n}\left\|y_{n}-p\right\|^{2} \\
& \leq \alpha_{n}\left\|f x_{n}-p\right\|^{2}+\eta_{n}\left\|x_{n}-p\right\|^{2}+\delta_{n}\left\|x_{n}-p\right\|^{2}-\delta_{n} \gamma_{n}\left(1-\gamma_{n}\right) g\left(\left\|T x_{n}-x_{n}\right\|\right) \\
& \leq \alpha_{n}\left\|f x_{n}-p\right\|^{2}+\left(1-\alpha_{n}\right)\left\|x_{n}-p\right\|^{2}-\delta_{n} \gamma_{n}\left(1-\gamma_{n}\right) g\left(\left\|T x_{n}-x_{n}\right\|\right),
\end{aligned}
$$

which is reduced to the inequality

$$
\delta_{n} \gamma_{n}\left(1-\gamma_{n}\right) g\left(\left\|T x_{n}-x_{n}\right\|\right) \leq \alpha_{n}\left(\left\|f x_{n}-p\right\|^{2}-\left\|x_{n}-p\right\|^{2}\right)+\left(\Gamma_{n}^{2}-\Gamma_{n+1}^{2}\right) .
$$

Then, by using the conditions (1)-(3) and the assumption $\Gamma_{n+1} \leq \Gamma_{n}$, we derive 
that

$$
\lim _{n \rightarrow \infty} g\left(\left\|T x_{n}-x_{n}\right\|\right)=0 .
$$

It follows from the property of $g$ that

$$
\lim _{n \rightarrow \infty}\left\|T x_{n}-x_{n}\right\|=0 \text {. }
$$

Repeat the argument for (3.4) to obtain

$$
\begin{aligned}
& \left\|x_{n+1}-p\right\|^{2} \\
& =\left\|\alpha_{n}\left(f x_{n}-p\right)+\delta_{n}\left(T z_{n}-p\right)+\eta_{n}\left(T y_{n}-p\right)\right\|^{2} \\
& \leq \alpha_{n}\left\|f x_{n}-p\right\|^{2}+\delta_{n}\left\|T z_{n}-p\right\|^{2}+\eta_{n}\left\|T y_{n}-p\right\|^{2}-\delta_{n} \eta_{n} g\left(\left\|T y_{n}-T z_{n}\right\|\right) \\
& \leq \alpha_{n}\left\|f x_{n}-p\right\|^{2}+\delta_{n}\left\|x_{n}-p\right\|^{2}+\eta_{n}\left\|x_{n}-p\right\|^{2}-\delta_{n} \eta_{n} g\left(\left\|T y_{n}-T z_{n}\right\|\right) \\
& =\alpha_{n}\left\|f x_{n}-p\right\|^{2}+\left(1-\alpha_{n}\right)\left\|x_{n}-p\right\|^{2}-\delta_{n} \eta_{n} g\left(\left\|T y_{n}-T z_{n}\right\|\right),
\end{aligned}
$$

which implies that

$$
\delta_{n} \eta_{n} g\left(\left\|T y_{n}-T z_{n}\right\|\right) \leq \alpha_{n}\left(\left\|f x_{n}-p\right\|^{2}-\left\|x_{n}-p\right\|^{2}\right)+\left(\Gamma_{n}^{2}-\Gamma_{n+1}^{2}\right) .
$$

Hence, by using the conditions (1)-(3), the assumption $\Gamma_{n+1} \leq \Gamma_{n}$ and the property of $g$, we derive that

$$
\lim _{n \rightarrow \infty}\left\|T y_{n}-T z_{n}\right\|=0 .
$$

Write

$$
x_{n+1}-T y_{n}=\alpha_{n}\left(f x_{n}-T y_{n}\right)+\delta_{n}\left(T z_{n}-T y_{n}\right)
$$

and apply the condition (1) and (3.9) to get

$$
\lim _{n \rightarrow \infty}\left\|x_{n+1}-T y_{n}\right\|=0 \text {. }
$$

Note that

$$
\begin{aligned}
& \left\|x_{n+1}-x_{n}\right\| \\
& \leq\left\|x_{n+1}-T y_{n}\right\|+\left\|T y_{n}-x_{n}\right\| \\
& \leq\left\|x_{n+1}-T y_{n}\right\|+\mu\left\|x_{n}-T x_{n}\right\|+\left\|x_{n}-y_{n}\right\| \\
& \leq\left\|x_{n+1}-T y_{n}\right\|+\mu\left\|x_{n}-T x_{n}\right\|+\beta_{n}\left\|x_{n}-T x_{n}\right\| .
\end{aligned}
$$

Apply the condition (1), (3.6) and (3.10) to get

$$
\lim _{n \rightarrow \infty}\left\|x_{n+1}-x_{n}\right\|=0
$$

Since $\left\{x_{n}\right\}$ is bounded, there exists a subsequence $\left\{x_{n_{k}}\right\}$ of $\left\{x_{n}\right\}$ such that $x_{n_{k}}$ converges weakly to a point $q$ and moreover

$$
\limsup _{n \rightarrow \infty}\left\langle f(p)-p, j\left(x_{n+1}-p\right)\right\rangle=\lim _{k \rightarrow \infty}\left\langle f(p)-p, j\left(x_{n_{k}+1}-p\right)\right\rangle .
$$

Apply (3.6), (3.12) and Lemma 2.4 to infer that $x_{n_{k}+1}$ converges weakly to a point $q$ and $q \in \operatorname{Fix}(T)$. This together with the property of the sunny nonexpansive retraction implies 


$$
\begin{aligned}
& \limsup _{n \rightarrow \infty}\left\langle f(p)-p, j\left(x_{n+1}-p\right)\right\rangle \\
& =\lim _{k \rightarrow \infty}\left\langle f(p)-p, j\left(x_{n_{k}+1}-p\right)\right\rangle \\
& =\lim _{k \rightarrow \infty}\langle f(p)-p, j(q-p)\rangle \\
& =\left\langle f(p)-P_{F(T)} p, j\left(q-P_{F(T)} p\right)\right\rangle \\
& \leq 0 .
\end{aligned}
$$

Finally, we prove that $\lim _{n \rightarrow \infty}\left\|x_{n}-p\right\|=0$. Using (3.1) and the assumption $\Gamma_{n+1} \leq \Gamma_{n}$, we have that, for all $n \geq n_{0}$,

$$
\begin{aligned}
\left\|x_{n+1}-p\right\|^{2} \\
=\alpha_{n}\left\langle f x_{n}-p, j\left(x_{n+1}-p\right)\right\rangle+\delta_{n}\left\langle T z_{n}-p, j\left(x_{n+1}-p\right)\right\rangle \\
\quad+\eta_{n}\left\langle T y_{n}-p, j\left(x_{n+1}-p\right)\right\rangle \\
\leq \alpha_{n}\left\langle f x_{n}-f(p), j\left(x_{n+1}-p\right)\right\rangle+\alpha_{n}\left\langle f(p)-p, j\left(x_{n+1}-p\right)\right\rangle \\
\quad+\delta_{n}\left\langle T z_{n}-p, j\left(x_{n+1}-p\right)\right\rangle+\eta_{n}\left\langle T y_{n}-p, j\left(x_{n+1}-p\right)\right\rangle \\
\leq \alpha_{n} r\left\|x_{n}-p\right\|\left\|x_{n+1}-p\right\|+\alpha_{n}\left\langle f(p)-p, j\left(x_{n+1}-p\right)\right\rangle \\
\quad+\delta_{n}\left\|T z_{n}-p\right\|\left\|x_{n+1}-p\right\|+\eta_{n}\left\|T y_{n}-p\right\|\left\|x_{n+1}-p\right\| \\
\leq \alpha_{n} r\left\|x_{n}-p\right\|\left\|x_{n+1}-p\right\|+\alpha_{n}\left\langle f(p)-p, j\left(x_{n+1}-p\right)\right\rangle \\
\quad+\delta_{n}\left\|z_{n}-p\right\|\left\|x_{n+1}-p\right\|+\eta_{n}\left\|y_{n}-p\right\|\left\|x_{n+1}-p\right\| \\
\leq \alpha_{n} r\left\|x_{n}-p\right\|\left\|x_{n+1}-p\right\|+\alpha_{n}\left\langle f(p)-p, j\left(x_{n+1}-p\right)\right\rangle \\
\quad+\delta_{n}\left\|x_{n}-p\right\|\left\|x_{n+1}-p\right\|+\eta_{n}\left\|x_{n}-p\right\|\left\|x_{n+1}-p\right\| \\
\leq\left(1-\alpha_{n}(1-r)\right)\left\|x_{n}-p\right\|\left\|x_{n+1}-p\right\|+\alpha_{n}\left\langle f(p)-p, j\left(x_{n+1}-p\right)\right\rangle \\
\leq\left(1-\alpha_{n}(1-r)\right)\left\|x_{n}-p\right\|^{2}+\alpha_{n}\left\langle f(p)-p, j\left(x_{n+1}-p\right)\right\rangle .
\end{aligned}
$$

By virtue of (3.13) and Lemma 2.7 and noticing (3.14), we get

$$
\lim _{n \rightarrow \infty}\left\|x_{n}-p\right\|=0 .
$$

Case B. Assume that $\left\{\Gamma_{n}\right\}$ is nondecreasing. From Lemma 2.8, there exists a nondecreasing sequence $\{\tau(n)\} \subset \mathbb{N}$ such that

$$
\max \left\{\Gamma_{\tau(n)}, \Gamma_{n}\right\} \leq \Gamma_{\tau(n)+1} .
$$

Following an argument similar to that in Case A and noticing (3.14), we derive that

$$
\lim _{n \rightarrow \infty}\left\|T x_{\tau(n)+1}-x_{\tau(n)}\right\|=0
$$

and

$$
\lim _{n \rightarrow \infty}\left\|x_{\tau(n)+1}-x_{\tau(n)}\right\|=0 .
$$

Repeat the argument for (3.13) to obtain

$$
\limsup _{n \rightarrow \infty}\left\langle f(p)-p, j\left(x_{\tau(n)+1}-p\right)\right\rangle \leq 0 .
$$

Finally, we show that $\lim _{n \rightarrow \infty} \Gamma_{\tau(n)}=0$. It follows (3.1) and (3.17) that 


$$
\begin{aligned}
\| & x_{\tau(n)+1}-p \|^{2} \\
= & \alpha_{n}\left\langle f x_{\tau(n)}-p, j\left(x_{\tau(n)+1}-p\right)\right\rangle+\delta_{\tau(n)}\left\langle T z_{\tau(n)}-p, j\left(x_{\tau(n)+1}-p\right)\right\rangle \\
& +\eta_{\tau(n)}\left\langle T y_{\tau(n)}-p, j\left(x_{\tau(n)+1}-p\right)\right\rangle \\
\leq & \alpha_{\tau(n)}\left\langle f x_{\tau(n)}-f(p), j\left(x_{\tau(n)+1}-p\right)\right\rangle+\alpha_{\tau(n)}\left\langle f(p)-p, j\left(x_{\tau(n)+1}-p\right)\right\rangle \\
& +\delta_{\tau(n)}\left\langle T z_{\tau(n)}-p, j\left(x_{\tau(n)+1}-p\right)\right\rangle+\eta_{\tau(n)}\left\langle T y_{\tau(n)}-p, j\left(x_{\tau(n)+1}-p\right)\right\rangle \\
\leq & \alpha_{\tau(n)} r\left\|x_{\tau(n)}-p\right\|\left\|x_{\tau(n)+1}-p\right\|+\alpha_{\tau(n)}\left\langle f(p)-p, j\left(x_{\tau(n)+1}-p\right)\right\rangle \\
& +\delta_{\tau(n)}\left\|T z_{\tau(n)}-p\right\|\left\|x_{\tau(n)+1}-p\right\|+\eta_{\tau(n)}\left\|T y_{\tau(n)}-p\right\|\left\|x_{\tau(n)+1}-p\right\| \\
\leq & \alpha_{\tau(n)} r\left\|x_{\tau(n)}-p\right\|\left\|x_{n+1}-p\right\|+\alpha_{\tau(n)}\left\langle f(p)-p, j\left(x_{\tau(n)+1}-p\right)\right\rangle \\
& +\delta_{\tau(n)}\left\|z_{\tau(n)}-p\right\|\left\|x_{n+1}-p\right\|+\eta_{\tau(n)}\left\|y_{\tau(n)}-p\right\|\left\|x_{n+1}-p\right\| \\
\leq & \alpha_{\tau(n)}\left\|x_{\tau(n)}-p\right\|\left\|x_{n+1}-p\right\|+\alpha_{\tau(n)}\left\langle f(p)-p, j\left(x_{\tau(n)+1}-p\right)\right\rangle \\
& +\delta_{\tau(n)}\left\|x_{\tau(n)}-p\right\|\left\|x_{n+1}-p\right\|+\eta_{\tau(n)}\left\|x_{\tau(n)}-p\right\|\left\|x_{n+1}-p\right\| \\
\leq & \left(1-\alpha_{\tau(n)}(1-r)\right)\left\|x_{\tau(n)}-p\right\|\left\|x_{n+1}-p\right\|+\alpha_{\tau(n)}\left\langle f(p)-p, j\left(x_{\tau(n)+1}-p\right)\right\rangle \\
\leq & \left(1-\alpha_{n}(1-r)\right)\left\|x_{\tau(n)+1}-p\right\|^{2}+\alpha_{n}\left\langle f(p)-p, j\left(x_{\tau(n)+1}-p\right)\right\rangle .
\end{aligned}
$$

After simplifying, we have

$$
(1-r)\left\|x_{\tau(n)+1}-p\right\|^{2} \leq\left\langle f(p)-p, j\left(x_{\tau(n)+1}-p\right)\right\rangle .
$$

This together with the (3.17) implies that

$$
\lim _{n \rightarrow \infty}\left\|x_{\tau(n)+1}-p\right\|=0,
$$

Further, Lemma 2.8 implies

$$
\lim _{n \rightarrow \infty}\left\|x_{n}-p\right\| \leq \lim _{n \rightarrow \infty}\left\|x_{\tau(n)+1}-p\right\|=0,
$$

that is, $x_{n} \rightarrow p$ as $n \rightarrow \infty$. This completes the proof.

Remark 3.2. The main results in this paper extend and generalize corresponding results in [7] [8] in the following senses.

1) The subset $C$ of Banach space $E$ does not have to be compact in our Theorem 3.1. However, this assumption is very necessary in Theorem 3.4 of Usurelu et al. [7] and Corollary2 of Houmani and Turcanu [8].

2) Our result is new and the proofs are simple and different from those in [7] [8].

\section{An Extension of Our Main Results}

From Theorem 3.1, we deduce immediately the following results

Corollary 4.1. Let $C$ be a nonempty closed convex subset of a uniformly convex and smooth Banach space $E$ which admits a weakly continuous duality mapping J, $f: C \rightarrow C$ be a contractive mapping with constant $r \in(0,1), T: C \rightarrow C$ be a 
nonexpansive mapping with $\operatorname{Fix}(T) \neq \varnothing$. For arbitrarily given $x_{0} \in C$, let $\left\{x_{n}\right\}$ be the sequence generated iteratively by:

$$
\left\{\begin{array}{l}
z_{n}=\left(1-\gamma_{n}\right) x_{n}+\gamma_{n} T x_{n}, \\
y_{n}=\left(1-\beta_{n}\right) x_{n}+\beta_{n} T x_{n}, \\
x_{n+1}=\alpha_{n} f x_{n}+\delta_{n} T z_{n}+\eta_{n} T y_{n}, \quad \forall n \in \mathbb{N},
\end{array}\right.
$$

where $\left\{\alpha_{n}\right\},\left\{\beta_{n}\right\},\left\{\gamma_{n}\right\},\left\{\delta_{n}\right\}$ and $\left\{\eta_{n}\right\}$ are real number sequences in $[0$, 1] satisfying:

1) $\lim _{n \rightarrow \infty} \alpha_{n}=0$ and $\sum_{n=1}^{\infty} \alpha_{n}=\infty$,

2) $\alpha_{n}+\delta_{n}+\eta_{n}=1$,

3) $0<\liminf _{n \rightarrow \infty} \delta_{n} \leq \limsup _{n \rightarrow \infty} \delta_{n}<1, \quad 0<\liminf _{n \rightarrow \infty} \gamma_{n} \leq \limsup _{n \rightarrow \infty} \gamma_{n}<1$ and $0<\liminf _{n \rightarrow \infty} \eta_{n} \leq \limsup _{n \rightarrow \infty} \eta_{n}<1$.

Then the sequence $\left\{x_{n}\right\}$ converges strongly to a point $p \in \operatorname{Fix}(T)$, which is also the unique solution of the hierarchical variational inequality

$$
\langle f(p)-p, j(q-p)\rangle \leq 0, \forall q \in \operatorname{Fix}(T) .
$$

In other words, $p$ is the unique fixed point of the mapping $P_{\mathrm{Fix}(T)} f$, that is, $p=P_{\mathrm{Fix}(T)} f(p)$.

Corollary 4.2. Let $C$ be a nonempty closed convex subset of a uniformly convex and smooth Banach space $E$ which admits a weakly continuous duality mapping J. Let $T: C \rightarrow C$ be a nonexpansive mapping with $\operatorname{Fix}(T) \neq \varnothing$. For arbitrarily given $x_{0}, u \in C$, let $\left\{x_{n}\right\}$ be the sequence generated iteratively by:

$$
\left\{\begin{array}{l}
z_{n}=\left(1-\gamma_{n}\right) x_{n}+\gamma_{n} T x_{n}, \\
y_{n}=\left(1-\beta_{n}\right) x_{n}+\beta_{n} T x_{n}, \\
x_{n+1}=\alpha_{n} u+\delta_{n} T z_{n}+\eta_{n} T y_{n}, \quad \forall n \in \mathbb{N},
\end{array}\right.
$$

where $\left\{\alpha_{n}\right\},\left\{\beta_{n}\right\},\left\{\gamma_{n}\right\},\left\{\delta_{n}\right\}$ and $\left\{\eta_{n}\right\}$ are real number sequences in $[0$, 1] satisfying.

1) $\lim _{n \rightarrow \infty} \alpha_{n}=0$ and $\sum_{n=1}^{\infty} \alpha_{n}=\infty$,

2) $\alpha_{n}+\delta_{n}+\eta_{n}=1$,

3) $0<\liminf _{n \rightarrow \infty} \delta_{n} \leq \limsup _{n \rightarrow \infty} \delta_{n}<1, \quad 0<\liminf _{n \rightarrow \infty} \gamma_{n} \leq \limsup _{n \rightarrow \infty} \gamma_{n}<1$ and $0<\liminf _{n \rightarrow \infty} \eta_{n} \leq \limsup _{n \rightarrow \infty} \eta_{n}<1$.

Then the sequence $\left\{x_{n}\right\}$ converges strongly to a point $p \in \operatorname{Fix}(T)$, which is also the unique solution of the hierarchical variational inequality

$$
\langle u-p, j(q-p)\rangle \leq 0, \quad \forall q \in \operatorname{Fix}(T) .
$$

In other words, $p$ is the unique fixed point of the mapping $P_{\operatorname{Fix}(T)} u$, that is, $p=P_{\operatorname{Fix}(T)} u$.

Corollary 4.3. Let $C$ be a nonempty closed convex subset of a Hilbert space $H$, $f: C \rightarrow C$ be a contractive mapping with constant $r \in(0,1), T: C \rightarrow C$ be a nonexpansive mapping with $\operatorname{Fix}(T) \neq \varnothing$. For arbitrarily given $x_{0} \in C$, let $\left\{x_{n}\right\}$ be the sequence generated iteratively by:

$$
\left\{\begin{array}{l}
z_{n}=\left(1-\gamma_{n}\right) x_{n}+\gamma_{n} T x_{n}, \\
y_{n}=\left(1-\beta_{n}\right) x_{n}+\beta_{n} T x_{n}, \\
x_{n+1}=\alpha_{n} f x_{n}+\delta_{n} T z_{n}+\eta_{n} T y_{n}, \quad \forall n \in \mathbb{N},
\end{array}\right.
$$


where $\left\{\alpha_{n}\right\},\left\{\beta_{n}\right\},\left\{\gamma_{n}\right\},\left\{\delta_{n}\right\}$ and $\left\{\eta_{n}\right\}$ are real number sequences in [0, 1] satisfying.

1) $\lim _{n \rightarrow \infty} \alpha_{n}=0$ and $\sum_{n=1}^{\infty} \alpha_{n}=\infty$,

2) $\alpha_{n}+\delta_{n}+\eta_{n}=1$,

3) $0<\liminf _{n \rightarrow \infty} \delta_{n} \leq \limsup _{n \rightarrow \infty} \delta_{n}<1, \quad 0<\liminf _{n \rightarrow \infty} \gamma_{n} \leq \limsup _{n \rightarrow \infty} \gamma_{n}<1$ and $0<\liminf _{n \rightarrow \infty} \eta_{n} \leq \limsup _{n \rightarrow \infty} \eta_{n}<1$.

Then the sequence $\left\{x_{n}\right\}$ converges strongly to a point $p \in \operatorname{Fix}(T)$, which is also the unique solution of the hierarchical variational inequality

$$
\langle f(p)-p, q-p\rangle \leq 0, \forall q \in \operatorname{Fix}(T) .
$$

In other words, $p$ is the unique fixed point of the mapping $P_{\operatorname{Fix}(T)} f$, that is, $p=P_{\operatorname{Fix}(T)} f(p)$.

\section{Conclusion}

The present work has been aimed to theoretically establish a new iterative scheme for finding a common element of the set of common fixed points of generalized nonexpansive mappings enriched with property (E) and the set of solutions of some variational inequalities in Banach spaces without the compactness assumption. Our results can be viewed as improvement, supplementation, development and extension of the corresponding results in some references to a great extent.

\section{Acknowledgements}

This research was supported by the Key Scientific Research Projects of Higher Education Institutions in Henan Province (20A110038).

\section{Conflicts of Interest}

The authors declare no conflicts of interest regarding the publication of this paper.

\section{References}

[1] Grillo, G. and Stampacchia, G. (1964) Formes bilineaires coercivities sur les ensembles convexes. Comptes Rendus de I Académie des Sciences, 258, 4413-4416.

[2] Qin, X., Chang, S.S., Cho, Y.J. and Kang, S.M. (2010) Approximation of Solutions to a System of Variational Inclusions in Banach Spaces. Journal of Inequalities and Applications, 2010, Article ID: 916806. https://doi.org/10.1155/2010/916806

[3] Yao, Y. and Yao, J. (2007) On Modified Iterative Method for Nonexpansive Mappings and Monotone Mappings. Applied Mathematics and Computation, 186, 1551-1558. https://doi.org/10.1016/j.amc.2006.08.062

[4] Brezis, H. (1968) Equations et inequations non lineaires dans les espaces vectoriels en dualite. Annales de I Institut Fourier (Grenoble), 18, 115-175. https://doi.org/10.5802/aif.280

[5] Moreau, J.J. (1965) Proximite et dualite dans un espaces hilbertien. Bulletin de la Société Mathématique de France, 93, 273-299. https://doi.org/10.24033/bsmf.1625 
[6] Garcia-Falset, J., Llorens-Fuster, E. and Suzuki, T. (2011) Fixed Point Theory for a Class of Generalized Nonexpansive Mappings. Journal of Mathematical Analysis and Applications, 375, 185-195. https://doi.org/10.1016/j.jmaa.2010.08.069

[7] Usurelu, G.I., Bejenaru, A. and Postolache, M. (2020) Operators with Property (E) as Concerns Numerical Analysis and Visualization. Numerical Functional Analysis and Optimization, 3, 1-22.

[8] Houmani, H. and Turcanu, T. (2019) CQ-Type Algorithm for Reckoning Best Proximity Points of EP-Operators. Symmetry, 12, 4. https://doi.org/10.3390/sym12010004

[9] Song, Y. and Ceng, L. (2015) Strong Convergence of a General Iterative Algorithm for a Finite Family of Accretive Operators in Banach Spaces. Fixed Point Theory and Applications, 2015, Article No. 90. https://doi.org/10.1186/s13663-015-0335-0

[10] Song, Y. and Chen, X.H. (2019) Algorithms for Common Solutions to Generalized Mixed Equilibrium Problems and Fixed Point Problems under Nonlinear Transformations in Banach Spaces. Journal of Applied Mathematics and Physics, 7, 2632-2649. https://doi.org/10.4236/jamp.2019.711179

[11] Chen, X.H., Song, Y., He, J.Y. and Gong, L.P. (2019) Self-Adaptive Algorithms for the Split Common Fixed Point Problem of the Demimetric Mappings. Journal of Applied Mathematics and Physics, 7, 2187-2199. https://doi.org/10.4236/jamp.2019.710150

[12] Yao, Y.H., Liou, Y.C. and Yao, J.C. (2015) Finding the Minimum Norm Common Element of Maximal Monotone Operators and Nonexpansive Mappings without Involving Projection. Journal of Nonlinear and Convex Analysis, 16, 835-854.

[13] Xu, H.K. (2010) Iterative Methods for the Split Feasibility Problem in Infinite-Dimensional Hilbert Spaces. Inverse Problems, 26, Article ID: 105018. https://doi.org/10.1088/0266-5611/26/10/105018

[14] Brower, F.E. (1967) Convergence Theorems for Sequences of Nonlinear Mappings in Banach Spaces. Mathematische Zeitschrift, 100, 201-225. https://doi.org/10.1007/BF01109805

[15] Gossez, J.P. and Lami Dozo, E. (1972) Some Geometric Properties Related to the Fixed Point Theory for Nonexpansive Mappings. Pacific Journal of Mathematics, 40, 565-573. https://doi.org/10.2140/pjm.1972.40.565

[16] Sitthithakerngkiet, K., Song, Y. and Kumam, P. (2019) An Algorithm for Solving a Class of Accretive Variational Inequalities Involving Pseudo-Contractions. Journal of Fixed Point Theory and Applications, 21, 75. https://doi.org/10.1007/s11784-019-0708-9

[17] Beauzamy, B. (1982) Introduction to Banach Spaces and Their Geometry, I Mathematics Studies. North-Holland, Amsterdam.

[18] Reich, S. (1973) Asymptotic Behavior of Contractions in Banach Spaces. Journal of Mathematical Analysis and Applications, 44, 57-70. https://doi.org/10.1016/0022-247X(73)90024-3

[19] Song, Y.L. and Ceng, L.C. (2013) A General Iteration Scheme for Variational Inequality Problem and Common Fixed Point Problems of Nonexpansive Mappings in q-Uniformly Smooth Banach Spaces. Journal of Global Optimization, 57, 1327-1348. https://doi.org/10.1007/s10898-012-9990-4

[20] Chang, S.S., Kim, J.K. and Wang, X.R. (2010) Modified Block Iterative Algorithm for Solving Convex Feasibility Problems in Banach Spaces. Journal of Inequalities and Applications, 2010, Article ID: 869684. https://doi.org/10.1155/2010/869684 
[21] Xu, H.K. (2002) Iterative Algorithms for Nonlinear Operators. Journal of the London Mathematical Society, 66, 240-256. https://doi.org/10.1112/S0024610702003332

[22] Mainge, P.E. (2007) Approximation Methods for Common Fixed Points of Nonexpansive Mappings in Hilbert Spaces. Journal of Mathematical Analysis and Applications, 325, 469-479. https://doi.org/10.1016/j.jmaa.2005.12.066 